\title{
Long non-coding RNA DLEU1 promotes cell proliferation of glioblastoma multiforme
}

\author{
JIANCUN WANG, XINGYUN QUAN, DINGTING PENG and GUANCHENG HU \\ Department of Neurosurgery, People's Hospital of Zhangjiajie, Zhangjiajie, Hunan 427000, P.R. China
}

Received June 4, 2018; Accepted January 10, 2019

DOI: $10.3892 / \mathrm{mmr} .2019 .10428$

\begin{abstract}
Glioblastoma multiforme (GBM) is the most common malignant tumor with high morbidity and mortality. This study investigated the role of long non-coding RNAs (lncRNAs) in glioblastomagenesis progression. Using the GSE2223 and GSE59612 datasets, and RNA sequencing data of GBM from The Cancer Genome Atlas, differentially expressed (DE) genes including DE messenger RNAs (DEmRNAs) and DElncRNAs between GBM and normal controls were identified. Based on the competing endogenous RNA hypothesis, DElncRNA-micro RNA (miRNA)-DEmRNA interactions were obtained by target gene prediction. Gene Ontology (GO) and Kyoto Encyclopedia of Gene and Genomes pathway analysis of DEmRNAs in the DElncRNA-miRNA-DEmRNA network was performed. Expression and function analyses of DElncRNAs were performed by reverse transcription-polymerase chain reaction (RT-PCR) and an established viability assay, respectively. In total, $712 \mathrm{DE}$ genes were identified. Significant upregulation of lncRNA deleted in lymphocytic leukemia 1 (DLEU1) was revealed in GBM and a number of other types of cancer. DLEU1 interacted with 315 miRNAs and 105 DEmRNAs. The DEmRNAs were mainly enriched in tumorigenesis-associated GO terms (angiogenesis, positive regulation of cell proliferation, positive regulation of fibroblast apoptotic processes and regulation of neutrophil migration) and pathways (Hippo signaling pathway, cancer pathways, and Wnt signaling pathway). Correlation analysis revealed that mRNA TNF receptor associated factor 4 (TRAF4) was associated with DLEU1 expression. RT-PCR demonstrated that the expression levels of DLEU1 and TRAF4 were increased in GBM tissues. Small interfering RNA demonstrated that silencing DLEU1 downregulated TRAF4. The viability of GBM cells was significantly decreased following RNA interference with DLEU1 and TRAF4 production. The results demonstrate that
\end{abstract}

Correspondence to: Mr. Guancheng $\mathrm{Hu}$, Department of Neurosurgery, People's Hospital of Zhangjiajie, 192 Guyong Road, Yongding, Zhangjiajie, Hunan 427000, P.R. China

E-mail: guanchengHu1@yeah.net

Key words: lncRNA, glioblastoma, deleted in lymphocytic leukemia 1, tumorigenesis, cell proliferation
DLEU1 and TRAF4 is highly expressed in GBM tissues and promotes proliferation of GBM cells. It may act as a competing endogenous RNA and influence tumorigenesis of GBM.

\section{Introduction}

Gliomas are the most common primary brain tumors. They arise from cancerous brain and spinal cord glial cells $(1,2)$. Glioblastoma multiforme (GBM) is a World Health Organization grade IV glioma (3). It accounts for $56.1 \%$ of all gliomas and has a five-year survival rate of only $5.5 \%$ (4). Despite a number of treatment methods, including chemotherapeutic, radiological and surgical interventions, there has been no significant change in the therapeutic effect. Novel therapeutic approaches that are more effective are needed and require more knowledge of the pathogenesis of GBM.

Long non-coding RNA (lncRNA) is a type of RNA that contains over 200 nucleotides and that does not encode protein $(5,6)$. LncRNAs serve important roles in human diseases, especially involving tumors $(7,8)$. In gliomas, lncRNAs are involved in the regulation of various biological processes (9). For example, IncRNA nuclear paraspeckle assembly transcript 1 (NEAT1) is highly expressed in GBM tissues and perturbed expression of NEAT1 suppresses cell proliferation and invasion (10). High expression of lncRNA H19 promotes cell migration and enhances angiogenesis $(11,12)$. The expression of HOX transcript antisense RNA lncRNA is increased in GBM and is significantly associated with high grade brain tumors (13). Additionally, as competitive endogenous RNAs (ceRNAs), IncRNAs and messenger RNAs (mRNAs) can regulate one other by competing for the shared microRNAs (miRNAs/miRs) $(14,15)$. LncHERG is an lncRNA that acts as a competing RNA of miR-940. When IncHERG is highly expressed, cell multiplication, invasion and migration are inhibited in GBM (16).

In this study, the gene expression profile in GBM was analyzed using a microarray dataset and RNA-sequencing (RNA-Seq) datasets. Key lncRNAs in GBM were screened. Based on the ceRNA hypothesis (15), the lncRNA-miRNA-mRNA network was constructed and Gene Ontology (GO) and Kyoto Encyclopedia of Gene and Genomes (KEGG) pathway analysis of mRNAs in the network were performed. Reverse transcription-quantitative polymerase chain reaction (RT-qPCR) and cell proliferation 
assay were used to explore the functional role of the key lncRNAs in GBM.

\section{Materials and methods}

Data, sample and cell information. Gene expression profile datasets GSE2223 $(17,18)$ and GSE59612 (19) were obtained from the Gene Expression Omnibus database (https://www. ncbi.nlm.nih.gov/geo/) (20). Gene expression profile of GBM determined by RNA-Seq of the Cancer Genome Atlas (TCGA-GBM) was extracted from the Genomic Data Commons Data Portal (https://portal.gdc.cancer.gov/). The GSE2223 dataset included microarray data of 54 samples. Of these, four GBM adjacent normal brain tissues and 27 GBM tissues were included in the present study. A total of 17 GBM adjacent normal brain tissues and 39 GBM tissues of 92 samples in the GSE59612 dataset were selected in the present study. For the TCGA dataset, five GBM adjacent normal brain tissues and 156 GBM tissues were included.

A total of 10 GBM tissues were collected from GBM patients who were treated surgically at the People's Hospital of Zhangjiajie (Zhangjiajie, China) between April 2015 and March 2017. The 10 GBM adjacent normal brain tissues were obtained from patients with head trauma who underwent surgical treatment in the same hospital. The age of the partici-

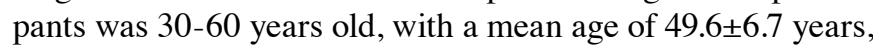
and the male:female ratio was 8:6. All samples were collected immediately following surgical resection and frozen rapidly in liquid nitrogen at $-70^{\circ} \mathrm{C}$. The experimental study was approved by the hospital's ethics committee and written informed consent was obtained from all participants.

The SHG-44 and U251 GBM cell lines were obtained from the American Type Culture Collection (ATCC; Manassas, VA, USA) and maintained in RPMI 1640 medium (Thermo Fisher Scientific, Inc., Waltham, MA, USA) containing 10\% fetal bovine serum (GE Healthcare Life Sciences, Logan, UT, USA), penicillin (100 units/ml), and streptomycin $(100 \mathrm{ug} / \mathrm{ml})$ and incubated in a $5 \% \mathrm{CO}_{2}$ incubator at $37^{\circ} \mathrm{C}$.

Small interfering si-NC, (si)-DLEU1 and si-tumor necrosis factor receptor-associated factor 4 (TRAF4) was purchased from Shanghai GenePharma Co., Ltd. (Shanghai, China). The si-NC, si-DLEU1, si-TRAF4 and the empty vectors (10 nM) were transfected into SHG-44 and U251 cell line using Lipofectamine ${ }^{\circledR} 2000$ (Invitrogen; Thermo Fisher Scientific, Inc.). Silencing was confirmed $12 \mathrm{~h}$ after transfection, by reverse transcription-polymerase chain reaction (RT-PCR) and western blot analysis. The specific primer sequences are presented in Table I.

Data analyses. Differentially expressed genes (DEGs) including differentially expressed mRNAs (DEmRNAs) and differentially expressed lncRNAs (DElncRNAs) between GBM and GBM adjacent normal brain tissues of the GSE2223 and GSE59612 datasets with normalized expression were analyzed using the Limmapackage(version 3.34.6; http://www. bioconductor.org/packages/release/bioc/html/limma.html). DEGs between GBM and GBM adjacent normal brain tissues of the TCGA-GBM were screened using the DESeq package (version 3.34.6, http://www.bioconductor. org/packages/release/bioc/html/DESeq.html). Count data were used to normalize data in the DESeq analysis. The fold-change (FC) of the DEGs, $\log _{2} \mathrm{FC}$ and false discovery rate (FDR) were obtained following testing. The cutoff thresholds were set to a $\mid \log _{2} \mathrm{FCl} \geq 1$ and FDR $<0.05$. The overlapping genes of the three datasets and genes displaying consistent regulation were included. Following annotation with Blast2GO5.0 (https://www.blast2go.com/), the lncRNA gene-type DEGs were selected as candidate lncRNAs.

Identification of cancer-associated lncRNAs. Gene Expression Profiling Interactive Analysis (GEPIA; http://gepia.cancerpku.cn/) was used to analyze expression levels, survival analysis and correlation of genes between tumors, and corresponding normal tissues. The expression levels of candidate lncRNAs between cancer and normal control were analyzed on the GEPIA website, which demonstrated 31 tumors types which have been tested. The parameters for expression levels screening are P-value Cutoff (0.01) and $\log 2 \mathrm{FC}$ Cutoff (1) on this site.

Construction of lncRNA-miRNA-mRNA network and correlation analysis of DElncRNAs and DEmRNAs. The integrated miRNA-DEmRNA and miRNA-DElncRNA pairs were simultaneously predicted by miRanda (http://www.microrna. org/microrna/home.do) and RNAhybrid (https://bibiserv. cebitec.uni-bielefeld.de/rnahybrid/), respectively. The parameters were set to energy $<-20$ and score $>150$ in miRanda and energy <-25 in RNAhybrid. Only the overlap results of miRanda and RNAhybrid were selected as the miRNA-DEmRNA and miRNA-DElncRNA pairs. The DElncRNA-miRNA-DEmRNA networks were constructed by miRNA-bridges using Cytoscape software (version 3.4.0) (21,22). Correlation analysis of DElncRNAs and DEmRNAs was performed using the GEPIA website.

GO and KEGG pathway analysis. To investigate the underlying functional role of lncRNAs, GO biological processes and KEGG pathway analysis were performed for the mRNAs in IncRNA-miRNA-mRNA interactions with DAVID 6.8 software (https://david.ncifcrf.gov/summary.jsp).

$R T$-PCR. Total RNA from GBM and normal brain samples was isolated using TRIzol reagent (Invitrogen; Thermo Fisher Scientific, Inc.) according to the manufacturer's protocol. After DNase digestion, RNA quantification and purity were measured by the ratio of $260 / 280 \mathrm{~nm}$. RNA integrity was measured by $1.2 \%$ agarose gel electrophoresis. The reverse transcriptase reaction kit (Applied Biosystems; Thermo Fisher Scientific, Inc.) was used to reverse-transcribe RNA samples at $50^{\circ} \mathrm{C}$ for $60 \mathrm{~min}$. RT-PCR was performed in triplicate according to the manufacturer's protocol of SYBR Green PCR Master Mix and reactions were carried using in a PCR Thermal Cycler (Takara Bio, Inc., Otsu, Japan). The conditions were: Initial denaturation at $95^{\circ} \mathrm{C}$ for $10 \mathrm{~min}$, followed by 40 cycles of denaturation at $95^{\circ} \mathrm{C}$ for $5 \mathrm{sec}$, and annealing and extension at $55-58^{\circ} \mathrm{C}$ for $30 \mathrm{sec}$. The relative expression levels of genes were calculated as relative quantification, calculated as $2^{-\Delta \Delta \mathrm{Cq}}$ (18).

Western blotting. Total proteins were extracted from cells using Radio Immunoprecipitation Assay Lysis Buffer (Beyotime Institute of Biotechnology, Shanghai, China). The 
Table I. Sequences for si-NC, si-DLEU1\#1, si-DLEU1\#2, si-TRAF4\#1 and si-TRAF4\#2.

\begin{tabular}{lcc}
\hline Name & \multicolumn{1}{c}{ Guide } & Passenger \\
\hline si-NC & 5'-UUCUCCGAACGUGUCACGUTT-3' & 5'-ACGUGACACGUUCGGAGAATT-3' \\
si-DLEU1\#1 & 5'-UUUUUUGUGCAGUUUCAGCAA-3' & 5'GCUGAAACUGCACAAAAAAUC-3' \\
si-DLEU1\#2 & 5'-UUCCUUUUUGAUAGUAUUCAA-3' & 5'GAAUACUAUCAAAAAGGAAAA-3' \\
si-TRAF4\#1 & 5'-UUAAUAAAUACAAUUCCGGAU-3' & 5'CCGGAAUUGUAUUUAUUAAUU-3' \\
si-TRAF4\#2 & 5'-UUUCAUAGGUGAAACGUGGAU-3' & 5'CCACGUUUCACCUAUGAAACA-3'
\end{tabular}

TRAF4, TNF receptor associated factor 4; DLEU1, deleted in lymphocytic leukemia 1; si, small interfering.

proteins were quantified using the bicinchoninic acid protein assay kit (Shanghai Solarbio Bioscience \& Technology Co., Ltd., Shanghai, China). Cell lysates were separated by $10 \%$ SDS-PAGE, transferred to polyvinylidene fluoride membranes, and membranes were blocked at room temperature with $5 \%$ skimmed milk in TSB Tween-20 $(0.05 \% \mathrm{v} / \mathrm{v}$; TBS-T $)$ for $1 \mathrm{~h}$ and incubated with specific antibodies at $4^{\circ} \mathrm{C}$ overnight. The primary antibody was a 1:1,000 dilution of rabbit anti-TRAF4 (cat. no. Ab108991; Abcam, Cambridge, UK) or mouse anti-GAPDH (cat. no. AG019; Beyotime Institute of Biotechnology). The membranes were washed three times for 10 min every time with TBS-T, followed by incubation with secondary antibodies goat anti-mouse immunoglobulin $(\mathrm{Ig}) \mathrm{G}$ (1:1,000; cat. no. A0216; Beyotime Institute of Biotechnology) and goat anti-rabbit IgG (1:2,000; cat. no. Ab6721; Abcam) for $1 \mathrm{~h}$ at room temperature. The intensities of the immunoreactivity were detected with an enhanced chemiluminescence kit (Bio-Rad Laboratories, Inc., Hercules, CA, USA). The images were developed on X-ray film. The experiments were repeated $\geq 3$ times.

Cell proliferation assay. A MTT kit (Sigma-Aldrich; Merck KGaA, Darmstadt, Germany) was used to analyze cell proliferation according to the manufacturer's protocol. All the cells were cultured in 96-well plates in the dark, the formazan was dissolved with dimethyl sulfoxide (DMSO) and the absorbance value at $570 \mathrm{~nm}$ was detected every $24 \mathrm{~h}$.

Statistical analyses. SPSS 17.0 software (SPSS, Inc., Chicago, IL, USA) was used to analyze statistical significance. All the experiments were independently performed three times. The data are expressed as the mean \pm standard deviation. The difference between the groups was analyzed with an analysis of variance (ANOVA) or Student's t test. Post hoc tests were performed using a Tukey test following the ANOVA. * and ${ }^{* *}$ refer to the statistically significant difference of expression $(\mathrm{P}<0.05)$ and extremely significant difference of expression $(\mathrm{P}<0.01)$, respectively. $\mathrm{P}<0.005$ was considered to indicate a statistically significant difference.

\section{Results}

Analysis of DEGs. A total of 730 overlapping DEGs were screened from the GSE2223, GSE59612 and TCGA-GBM datasets (Fig. 1A). Among these, 712 DEGs displaying consistent regulation in all the three datasets were selected for further study. The annotation analysis revealed three lncRNAs, 36 miscellaneous RNAs (miscRNAs) and 673 mRNAs (Fig. 1B). LncRNA DLEU1 was upregulated, while prostate androgen-regulated transcript 1 (PART1) and miR7-3HG were downregulated.

Identification of cancer-associated lncRNAs in GBM. Among the DElncRNAs, the expression of DLEU1 was upregulated in GBM and a number of other tumor types, including cervical squamous cell carcinoma and endocervical adenocarcinoma, colon adenocarcinoma, lymphoid neoplasm diffuse large B-cell lymphoma, brain lower grade glioma, lung squamous cell carcinoma, ovarian serous cystadenocarcinoma, pancreatic adenocarcinoma, rectum adenocarcinoma, stomach adenocarcinoma, thymoma, and uterine carcinosarcoma (Fig. 2). The results highlighted the important role of DLEU1 in carcinogenesis.

LncRNA-miRNA-mRNA network. The integrated DLEU1-miRNA-DEmRNA interactions were identified with the miRanda and RNAhybrid methods. The network was constructed using Cytoscape software (Fig. 3). In the lncRNA DLEU1-mediated ceRNA network, DLEU1 interacted with as many as 315 miRNAs and 105 DEmRNAs. Among the miRNAs, miR-107, miR-1179, miR-133a, miR-133b and miR-346 have been reported to be downregulated, and are involved in the progression of in GBM.

GO and pathway analysis. To investigate the functional role of genes in the DLEU1-miRNA-DEmRNA network, GO and pathway analysis of mRNAs were performed with DAVID 6.8 software $(23,24)$. The top $10 \mathrm{GO}$ terms and pathway terms are presented in Fig. 4. The mRNAs mainly enriched in the tumorigenesis associated GO terms were angiogenesis, positive regulation of cell proliferation, positive regulation of fibroblast apoptotic process, regulation of neutrophil migration and others. The pathway analysis revealed mRNAs primarily enriched in important pathways associated with tumorigenesis (Hippo signaling pathway, pathways in cancer and Wnt signaling pathway). The genes involved in the significant GO terms and pathway terms are presented in Table II. Wnt family member (WNT)5A, frizzled class receptor 7 (FZD7), transcription factor 7 like 1 (TCF7L1), WW domain containing transcription regulator 1 (WWTR1) and cluster of differentiation (CD)44 were enriched in at least four significant GO or pathway terms. 


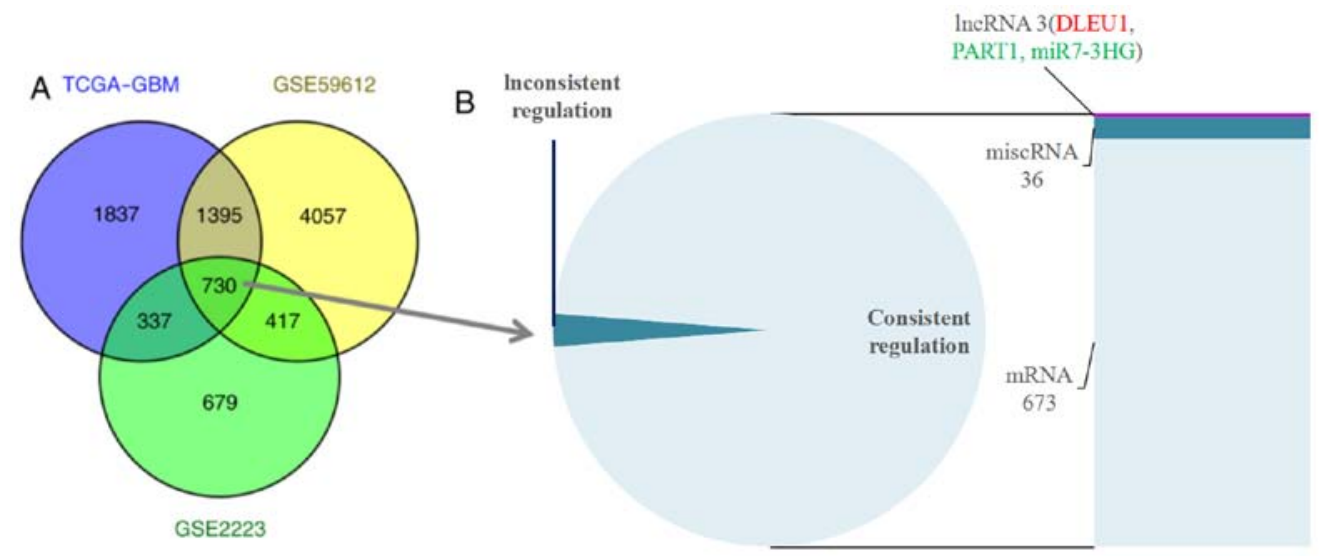

Figure 1. Overlapping DEGs of three datasets (GSE2223, GSE59612 and TCGA GBM RNA-Sequence). (A) Overlapping DEGs of three databases between normal and tumor samples. (B) The regulation association of the DEGs in the three datasets and the gene types of DEGs with a consistent regulation association. Inconsistent regulation is evident as inconsistent upregulation or downregulation of the DEGS in the three databases. DEG, differentially expressed genes, DEGs; TCGA, the cancer genome atlas; GBM, glioblastoma; lnc, long non-coding RNA; misc, miscellaneous.
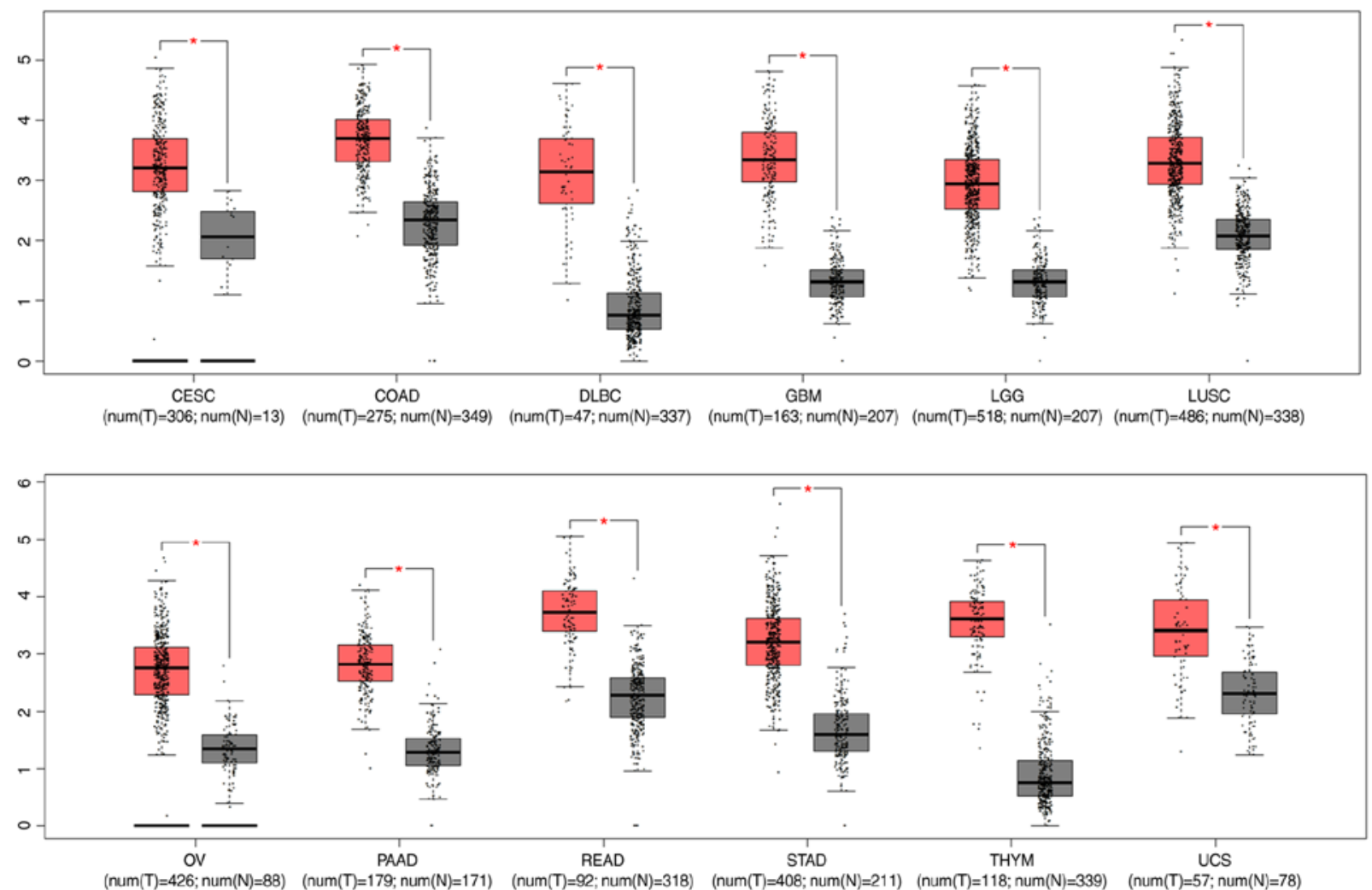

Figure 2. Expression level of lncRNA DLEU1 in multiple types of cancer. ${ }^{*} \mathrm{P}<0.05$. CESC, cervical squamous cell carcinoma and endocervical adenocarcinoma; COAD, colon adenocarcinoma; DLBC, lymphoid neoplasm diffuse large B-cell lymphoma; GBM, glioblastoma multiforme; LGG, brain lower grade glioma; LUSC, lung squamous cell carcinoma; OV, ovarian serous cystadenocarcinoma; PAAD, pancreatic adenocarcinoma; READ, rectum adenocarcinoma; STAD, stomach adenocarcinoma; THYM, thymoma; UCS, uterine carcinosarcoma; num, number; T, tumour; N, normal.

DLEU1 is positively associated with TRAF4. TRAF4 serves an important role in other cancers according to previous studies (25-28). Querying GEPIA revealed that the expression of TRAF4 in the DLEU1-miRNA-DEmRNA network was positively associated with DLEU1 (Fig. 5A). To validate the prediction, the relative expression levels of DLEU1 and DLEU1 were analyzed by RT-PCR in 10 and 10 GBM adjacent normal brain tissues. The expression levels of DLEU1 and TRAF4 were both significantly increased in GBM compared with normal control $(\mathrm{P}<0.01$; Fig. 5B and C).

Silencing DLEU1 downregulates TRAF4 and inhibits cell proliferation. The effects of silencing lncRNA were studied. Following silencing DLEU1 (Fig. 6A), cell viability decreased significantly in SHG-44 and U251 cell $(\mathrm{P}<0.01$; Fig. 6B). However, there was no significant difference between the negative control and blank control concerning 


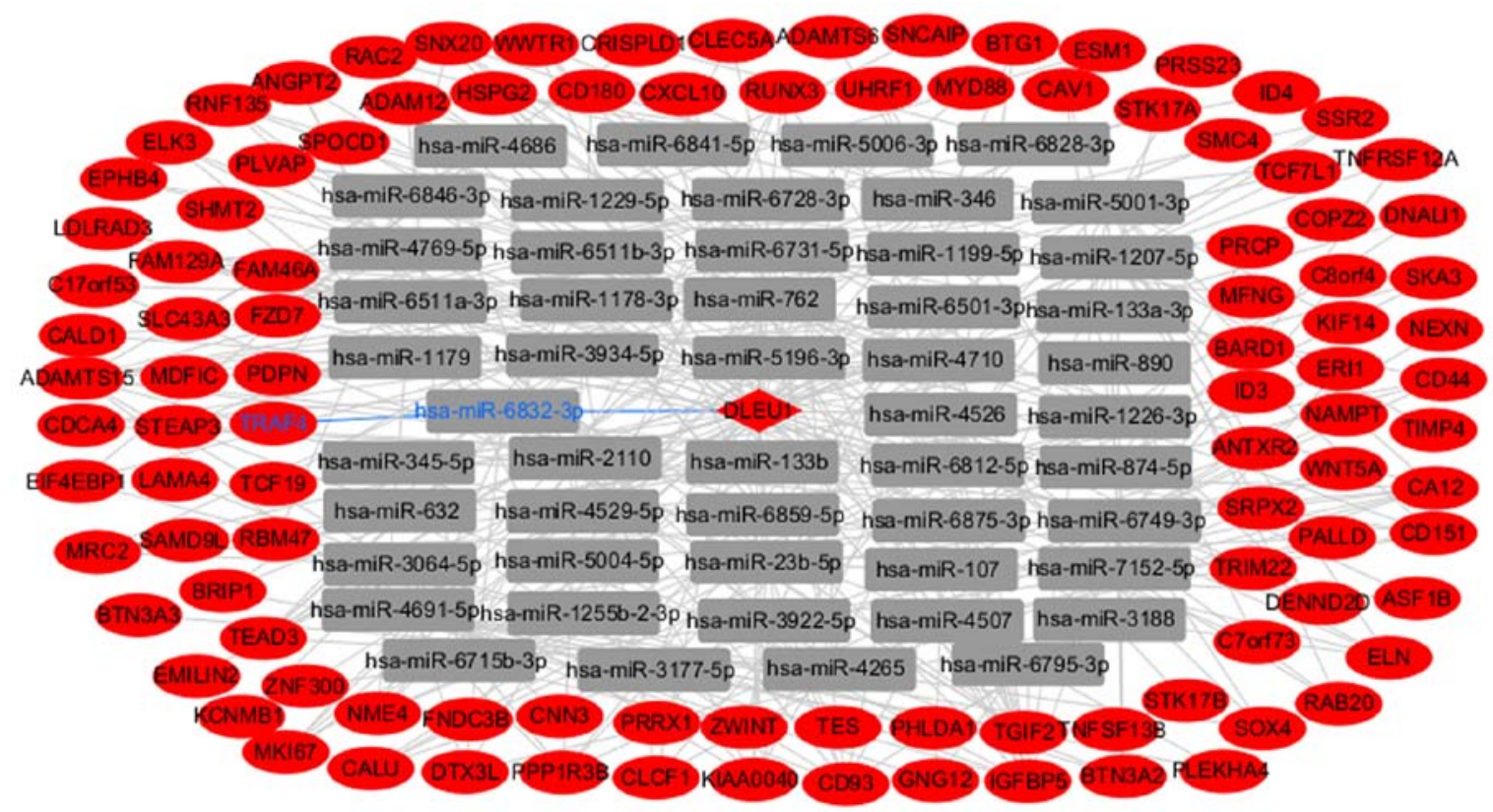

Figure 3. LncRNA DLEU1 mediates the lncRNA-miRNA-mRNA network. Diamonds represent lnRNA, round rectangles denote miRNA, and ovals represent mRNA. Red and gray denote upregulation and unknown regulation, respectively. miRNA/miR, microRNA; lnc, long non-coding RNA.

A

Go Analysis_BP

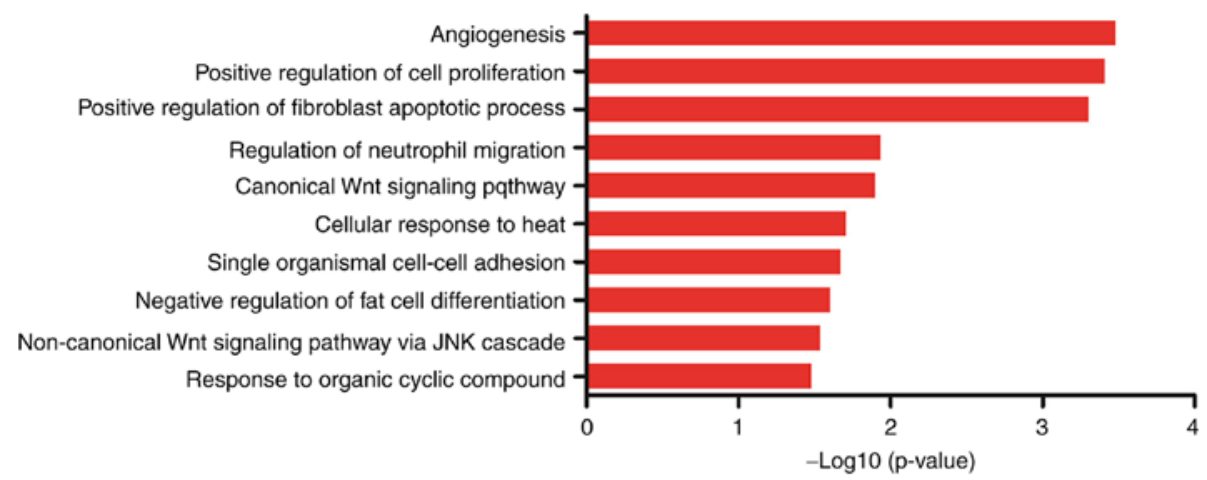

B

Pathway analysis

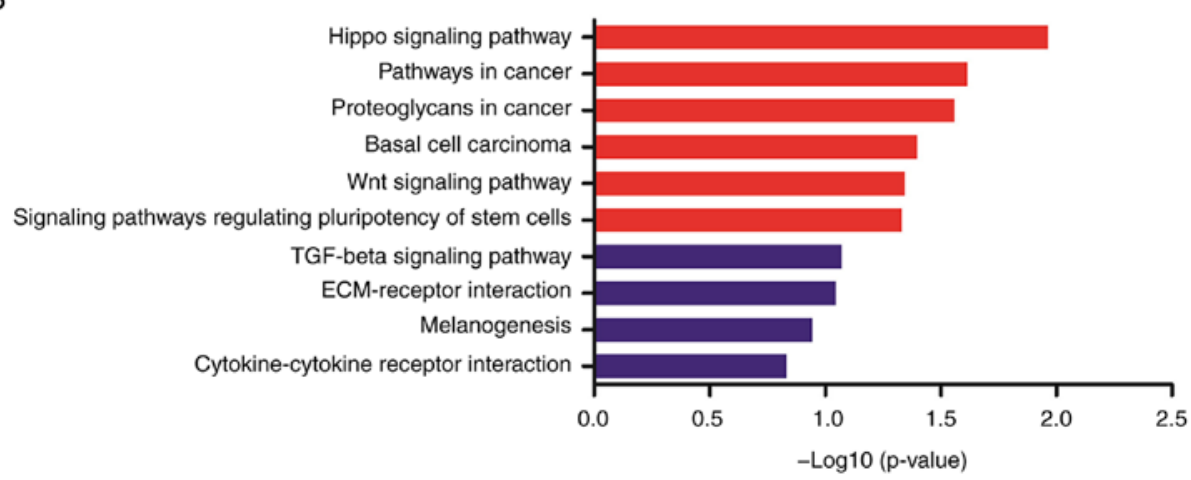

Figure 4. Gene Ontology and pathway analysis. (A) Gene Ontology and (B) pathway analysis of differentially expressed mRNAs in the lncRNA-miRNA-mRNA network. Red and blue denote $\mathrm{P}<0.05$ and $\mathrm{P}>0.05$, respectively. miRNA, microRNA; lnc, long non-coding RNA; TGF, transforming growth factor; ECM, extracellular matrix.

cell viability. Compared with the negative control transfected with the empty vector, the expression of TRAF4 was downregulated in SHG-44 and U251 cell transfected with si-DLEU1 at transcription (Fig. 6C) and the protein level (Fig. 6D).
Silencing TRAF4 inhibits cell proliferation. Similarly, the proliferation of cells was studied by silencing TRAF. Compared with the negative control transfected with the empty vector. After silencing TRAF4 (Fig. 6E), cell viability decreased significantly in SHG-44 and U251 cells $(\mathrm{P}<0.01$; 
Table II. Significant GO and pathway analysis of differentially expressed mRNAs in the DLEU1-miRNA-mRNAs network.

Terms

Genes

P-value

GO

Angiogenesis

Positive regulation of cell proliferation

Positive regulation of fibroblast apoptotic process

Regulation of neutrophil migration

Canonical Wnt signaling pathway

Cellular response to heat

Single organismal cell-cell adhesion

Negative regulation of fat cell differentiation

Non-canonical Wnt signaling pathway via JNK cascade

Response to organic cyclic compound

Mammary gland involution

Signal transduction

Negative regulation of protein export from nucleus

Kidney morphogenesis

$\mathrm{T}$ cell mediated immunity

Cartilage development

Positive regulation of protein binding

Pathway

Hippo signaling pathway

Pathways in cancer

Proteoglycans in cancer

Basal cell carcinoma

Wnt signaling pathway

Signaling pathways regulating pluripotency of stem cells
CAV1, SRPX2, TNFRSF12A, HSPG2, ESM1,

0.0003296

ELK3, ANGPT2, EPHB4

KIF14, NAMPT, SHMT2, TNFSF13B, RAC2,

CLCF1, SOX4, ID4, ESM1, WWTR1, CXCL10 0.0003889

BTG1, STK17B, STK17A

0.0004981

MYD88, RAC2

WNT5A, SOX4, TCF7L1, FZD7

MKI67, C8ORF4, CXCL10

0.0116385

0.0126456

0.0196642

SRPX2, CD44, CD93, PDPN

0.0212821

WNT5A, ID4, WWTR1

0.0249487

WNT5A, FZD7

0.0288453

NAMPT, MKI67, ANGPT2

0.0331943

CAV1, IGFBP5

0.0345148

NAMPT, PDPN, MRC2, GNG12, ELK3, CXCL10, 0.0375587 MYD88, RAC2, TNFSF13B, CLEC5A, ANGPT2,

TRAF4, IGFBP5

SOX4, BARD1

0.0401515

SOX4, WWTR1

0.0457556

BTN3A3, BTN3A2

0.0457556

WNT5A, CD44, PRRX1

0.0465266

WNT5A, MFNG, CAV1

0.0493935

WNT5A, TEAD3, WWTR1, TCF7L1, FZD7

0.010874

WNT5A, LAMA4, RAC2, GNG12, TCF7L1,

0.024194

FZD7, TRAF4

WNT5A, CAV1, CD44, HSPG2, FZD7

0.027611

WNT5A, TCF7L1, FZD7

0.040012

WNT5A, RAC2, TCF7L1, FZD7

0.045011

WNT5A, ID4, ID3, FZD7

0.046643

Genes in bold indicate these genes were enriched in at least four significant GO or pathway terms. GO, Gene Ontology; miRNA, microRNA; DLEU1, deleted in lymphocytic leukemia 1.
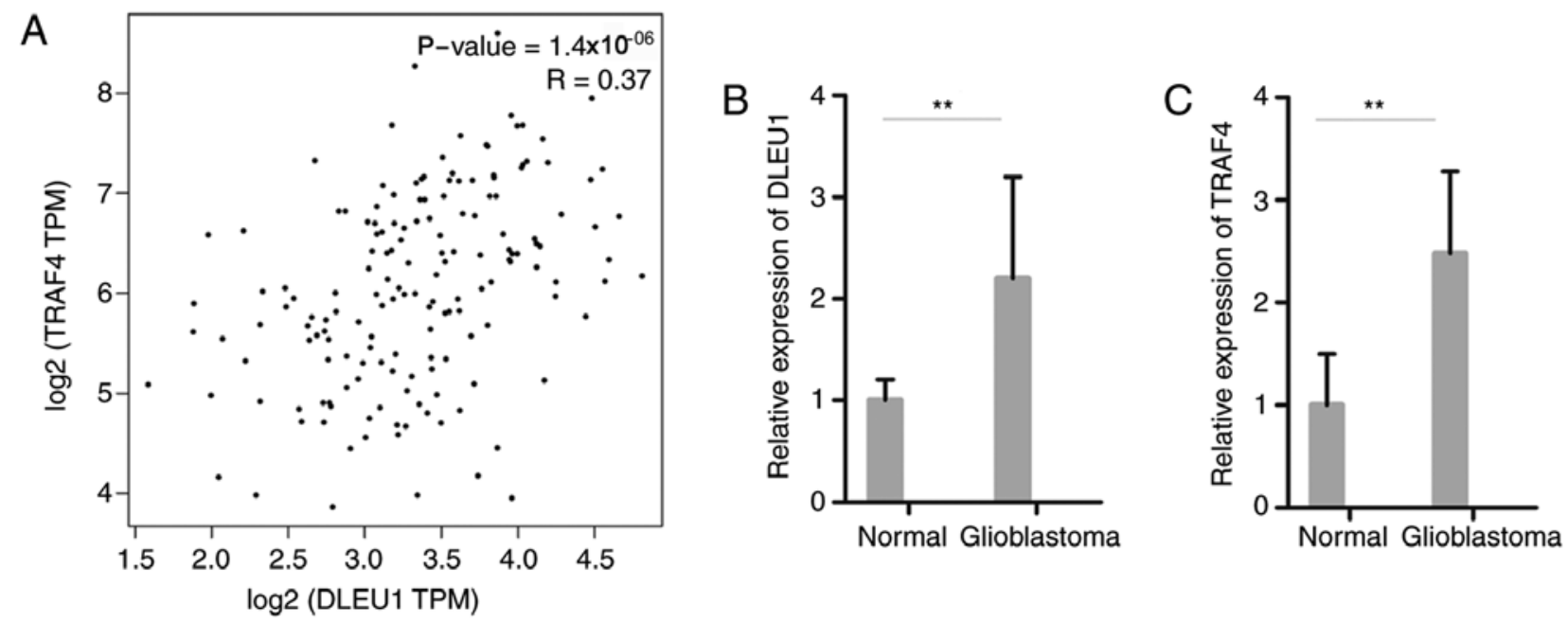

Figure 5. DLEU1 is positively associated with TRAF4. (A) Correlation analysis and relative expression analysis of lncRNA (B) DLEU1 and (C) TRAF4 in glioblastoma tissues. ${ }^{* *} \mathrm{P}<0.01, \mathrm{n}=3$. Lnc, long noncoding RNA; TRAF4, TNF receptor associated factor 4; DLEU1, deleted in lymphocytic leukemia 1. 
A

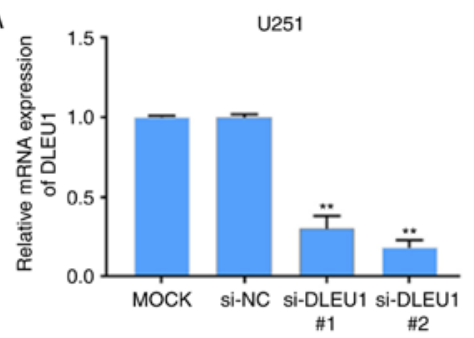

B

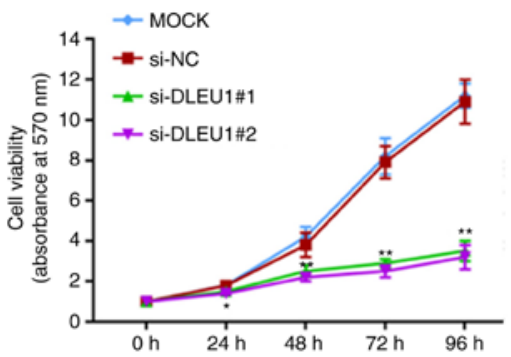

C

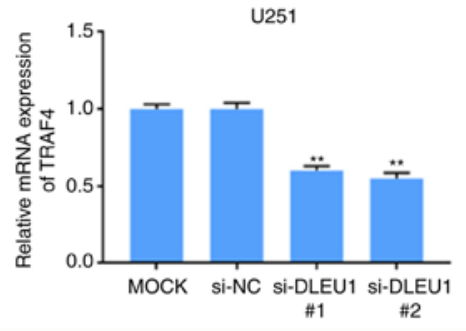

D
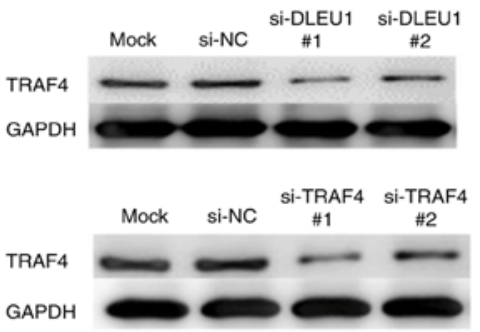

$\mathrm{E}$
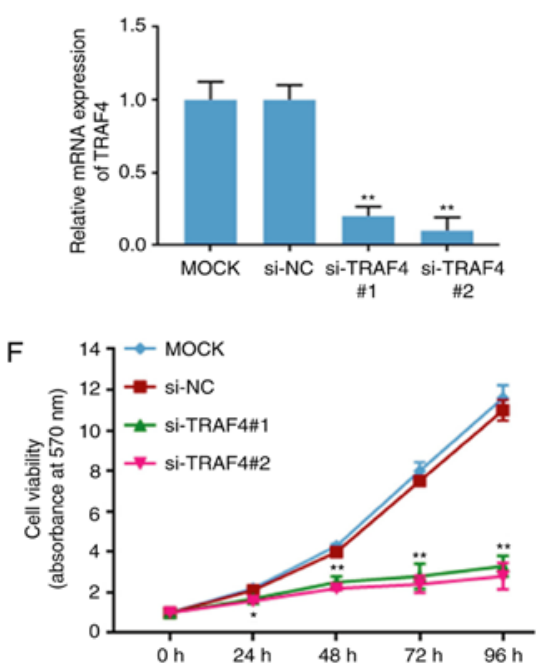

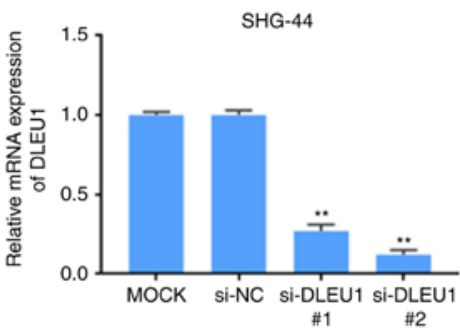

SHG-44

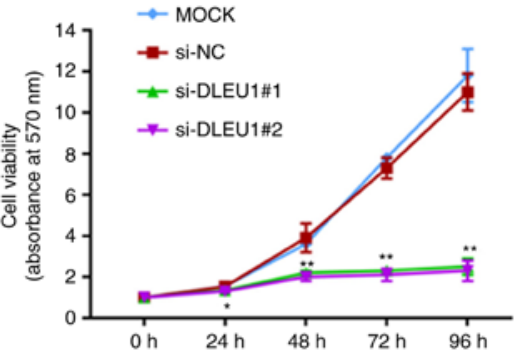

SHG-44

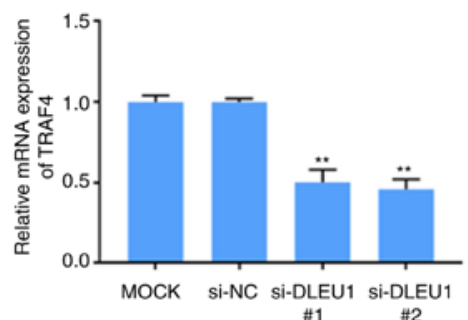

SHG-44
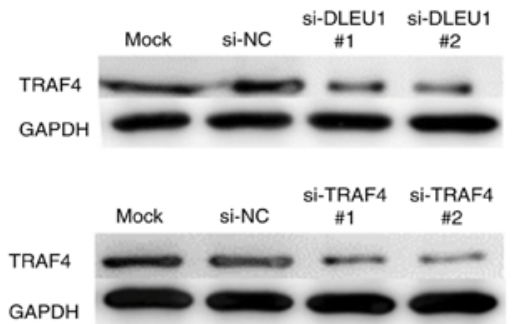

SHG-44
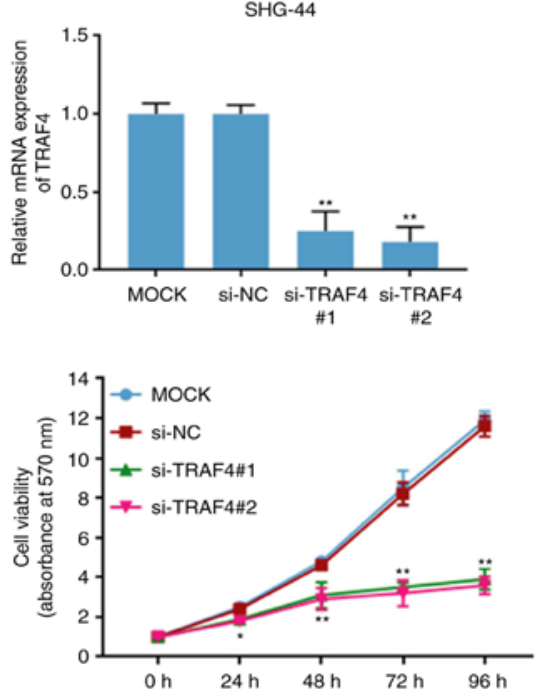

Figure 6. Silencing of 1ncRNA DLEU1 downregulates TRAF4 and inhibits cell proliferation. Silencing of lncRNA DLEU1 inhibited cell proliferation by MTT assays in (A) SHG-44 and (B) U251 cell. Silencing of lncRNA DLEU1 downregulates TRAF4 in SHG-44 and U251 cell transfected with si-DLEU1 at (C) the transcription and the (D) protein level. Silencing of TRAF4 inhibited cell proliferation as measured by MTT assays in (E) SHG-44 cells and (F) U251 cells. MOCK, SHG-44 cells and U251 cells transfected empty vector with no any other sequence; NC, SHG-44 cells and U251 cells transfected with non-targeting sequence in humans; si-DLEU1, SHG-44 cells and U251 cells transfected with si-DLEU1; si-TRAF4, SHG-44 cells and U251 cells transfected with si-TRAF4. ${ }^{*} \mathrm{P}<0.05$ and ${ }^{* *} \mathrm{P}<0.01, \mathrm{n}=3$. Si, small interfering; lnc, long non-coding; NC, negative control; TRAF4, TNF receptor associated factor 4; DLEU1, deleted in lymphocytic leukemia 1. 
Fig. 6F). However, there was no significant difference between the negative control and blank control concerning cell viability.

\section{Discussion}

In the present study, microarray data profiling and RNA-Seq profiles of GBM were integrated and re-analyzed. A total of 712 DEGs (673 DEmRNAs, 36 miscellaneous RNA and 3 DElncRNAs) were identified between GBM, and GBM adjacent normal brain tissues. Among the three lncRNAs (DLEU1, PART1 and miR7-3HG), DLEU1 was more expressed in a number of types of cancer, including GBM. The DLEU1-miRNA-DEmRNAs network was constructed. Several miRNAs in the DLEU1-miRNA-mRNAs network have been reported in the progression of tumorigenesis in GBM. For example, miR-107 is downregulated in glioma tissues and cell lines (29). Decreased expression of miRNA-107 promotes cell growth and invasion, inhibits cell apoptosis, and predicts a poor prognosis (30-32). miR-1179 and miR-346 (33) are downregulated in glioma tissues and cell lines, and their high expression inhibits cell proliferation. In GBM (34), miR-133a (35,36), miR-133b $(37,38)$ and miR-346 are downregulated in glioma tissues and cell lines, and high expression inhibits cell proliferation, migration, and invasion. The regulation of the association of these miRNAs in the authors' prediction was in concordance with these previous studies. Therefore, the analysis and prediction were credible.

DEmRNAs in the DLEU1-miRNA-mRNAs network were mainly enriched in the pathway terms of the Hippo signaling pathway, pathways in cancer and Wnt signaling pathway. The Hippo signaling pathway is reportedly a major signaling pathway that regulates cell proliferation and growth, and is important role in the tumorigenesis of GBM (39). CD44 was reported to be upregulated in GBM and protected cancer cells by attenuating the activation of the Hippo signaling pathway (40). Suppression of the activity of the Hippo signaling pathway using Amlexanox was reported to inhibit GBM cell proliferation and induce GBM cell apoptosis (41). The Wnt signaling pathway affects tumor initiation, cell migration and invasion of GBM (42). Multiple factors, including small molecules, including SEN461 (43), small non-coding RNAs, such as miRNA34a (44) and miRNA-577 (45), and mRNAs, such as zinc finger E-box binding homeobox 1 (46), WNT3A (47), and homeobox A13 (48), influence GBM progression via the Wnt signaling pathway. Presently, six DEmRNAs [WNT5A, TEA domain family members (TEAD)3, WWTR1, TCF7L1, FZD7 and Rac family small GTPase 2] in the DLEU1-miRNA-mRNA network were demonstrated to be enriched in the Hippo and Wnt signaling pathways. WNT5A induces GBM cell migration (49). WNT5A is increased in GBM tissues and overexpression of WNT5A promotes the differentiation, proliferation, migration, and invasive growth of GBM cells (49-51). WWTR1, also known as TAZ, is an important regulator of the Hippo signaling pathway. The interaction of WWTR1 with TEAD drives mesenchymal differentiation of malignant glioma and influences tumor progression (52). As a direct target, WWTR1 is upregulated in GBM and promotes cell proliferation (53). Finally, the high expression of FZD7 has been detected in GBM and is associated with poor survival $(54,55)$.
TRAF4 is overexpressed in tissues or cells in osteosarcoma (25), colon cancer (26), oral squamous cell carcinoma (27) and breast cancer (28). Presently, the knockdown of TRAF4 inhibited cell proliferation, migration and invasion, and induced apoptosis. A prior study reported the significantly high expression of DLEU1 in epithelial carcinoma, with associated promotion of cell multiplication, migration, and invasion and suppression of cell apoptosis (56). Other authors reported the intensified expression of DLEU1 in gastric cancer and the association with lymph node metastasis, tumor size, and advanced stage of pathology. Silencing of DLEU1 inhibited cell proliferation (57). In accordance with these findings, the expression level of DLEU1 and TRAF4 was confirmed to be high in GBM tissues by RT-PCR. The expression of DLEU1 was positively associated with TRAF4. RNA interference with DLEU1 downregulated TRAF and inhibited GBM cell proliferation. When TRAF4 knockdown occurs, cell proliferation is inhibited, with similar results to DLEU1. All results indicate that DLEU1 may affect the tumorigenesis of GBM by regulating the expression of TRAF4.

In conclusion, DLEU1 was intensified in GBM tissues and silencing of DLEU1 inhibited cell proliferation. Furthermore, miRNAs and mRNAs in the DLEU1-mediated ceRNA network are involved in cell differentiation, proliferation, migration, and invasive growth of GBM cells. These collective observations support the idea that DLEU1 may serve a pivotal role in the tumorigenesis of GBM. This study identifies a novel lncRNA that may act as a ceRNA in GBM. Further studies are needed to understand the molecular role of DLEU1 in GBM progression.

\section{Acknowledgements}

Not applicable.

\section{Funding}

The present study was funded by the Natural Science Foundation of Hunan Province (grant no. C2013-225).

\section{Availability of data materials}

All data generated or analyzed during this study are included in this published article.

\section{Authors' contributions}

GH and JW were responsible for the concept and design of the present study. JW and XQ acquired the data. JW and DP performed data analysis and experiments. JW drafted the article. All authors read and approved the final manuscript.

\section{Ethics approval and consent to participate}

The experimental study was approved by the ethics committee of People's Hospital of Zhangjiajie and written informed consent was obtained from all participants.

\section{Patient consent for publication}

Written informed consent was obtained from all participants. 


\section{Competing interests}

The authors declare that they have no competing interests.

\section{References}

1. Kohler BA, Ward E, McCarthy BJ, Schymura MJ, Ries LA Eheman C, Jemal A, Anderson RN, Ajani UA and Edwards BK Annual report to the nation on the status of cancer, 1975-2007, featuring tumors of the brain and other nervous system. J Natl Cancer Inst 103: 714-736, 2011.

2. Ohgaki H and Kleihues P: Epidemiology and etiology of gliomas Acta Neuropathol 109: 93-108, 2005.

3. Louis DN, Ohgaki H, Wiestler OD, Cavenee WK, Burger PC, Jouvet A, Scheithauer BW and Kleihues P: The 2007 WHO classification of tumours of the central nervous system. Acta Neuropathol 114: 97-109, 2007

4. Ostrom QT, Gittleman H, Liao P, Vecchione-Koval T, Wolinsky Y, Kruchko C and Barnholtz-Sloan JS: CBTRUS statistical report: Primary brain and other central nervous system tumors diagnosed in the United States in 2010-2014. Neuro Oncol 19: v1-v88, 2017.

5. Lipovich L. Johnson R and Lin CY: MacroRNA underdogs in a microRNA world: Evolutionary, regulatory, and biomedical significance of mammalian long non-protein-coding RNA Biochim Biophys Acta 1799: 597-615, 2010.

6. Mercer TR, Dinger ME and Mattick JS: Long non-coding RNAs: Insights into functions. Nat Rev Genet 10: 155-159, 2009.

7. Prensner JR and Chinnaiyan AM: The emergence of lncRNAs in cancer biology. Cancer Discov 1: 391-407, 2011.

8. Batista PJ and Chang HY: Long noncoding RNAs: Cellular address codes in development and disease. Cell 152: 1298-1307, 2013.

9. Wang $\mathrm{KC}$ and Chang HY: Molecular mechanisms of long noncoding RNAs. Mol Cell 43: 904-914, 2011.

10. Li M, Deng H, Peng H and Wang Q: Functional nanoparticles in targeting glioma diagnosis and therapies. J Nanosci Nanotechnol 14: 415-432, 2014.

11. Shi Y, Wang Y, Luan W, Wang P, Tao T, Zhang J, Qian J, Liu N and You Y: Long non-coding RNA H19 promotes glioma cell invasion by deriving miR-675. PLoS One 9: e86295, 2014.

12. Jiang X, Yan Y, Hu M, Chen X, Wang Y, Dai Y, Wu D, Wang Y, Zhuang $\mathrm{Z}$ and $\mathrm{Xia} \mathrm{H}$ : Increased level of H19 long noncoding RNA promotes invasion, angiogenesis, and stemness of glioblastoma cells. J Neurosurg 2016: 129-136, 2016.

13. Tan SK, Pastori C, Penas C, Komotar RJ, Ivan ME, Wahlestedt C and Ayad NG: Serum long noncoding RNA HOTAIR as a novel diagnostic and prognostic biomarker in glioblastoma multiforme. Mol Cancer 17: 74, 2018.

14. Qi X, Zhang DH, Wu N, Xiao JH, Wang X and Ma W: ceRNA in cancer: Possible functions and clinical implications. J Med Genet 52: 710-718, 2015.

15. Salmena L, Poliseno L, Tay Y, Kats L and Pandolfi PP: A ceRNA hypothesis: The Rosetta Stone of a hidden RNA language? Cell 146: 353-358, 2011.

16. Shi J, Wang YJ, Sun CR, Qin B, Zhang Y and Chen G: Long noncoding RNA lncHERG promotes cell proliferation, migration and invasion in glioblastoma. Oncotarget 8: 108031-108041, 2007.

17. Bredel M, Bredel C, Juric D, Duran GE, Yu RX, Harsh GR, Vogel H, Recht LD, Scheck AC and Sikic BI: Tumor necrosis factor-alpha-induced protein 3 as a putative regulator of nuclear factor-kappaB-mediated resistance to O6-alkylating agents in human glioblastomas. J Clin Oncol 24: 274-287, 2006.

18. Bredel M, Bredel C, Juric D, Harsh GR, Vogel H, Recht LD and Sikic BI: Functional network analysis reveals extended gliomagenesis pathway maps and three novel MYC-interacting genes in human gliomas. Cancer Res 65: 8679-8689, 2005.

19. Gill BJ, Pisapia DJ, Malone HR, Goldstein H, Lei L, Sonabend A Yun J, Samanamud J, Sims JS, Banu M, et al: MRI-localized biopsies reveal subtype-specific differences in molecular and cellular composition at the margins of glioblastoma. Proc Natl Acad Sci USA 111: 12550-12555, 2014.

20. Edgar R, Domrachev $M$ and Lash AE: Gene Expression Omnibus: NCBI gene expression and hybridization array data repository. Nucleic Acids Res 30: 207-210, 2002.
21. Bindea G, Mlecnik B, Hackl H, Charoentong P, Tosolini M, Kirilovsky A, Fridman WH, Pagès F, Trajanoski $\mathrm{Z}$ and Galon J: ClueGO: A Cytoscape plug-in to decipher functionally grouped gene ontology and pathway annotation networks. Bioinformatics 25: 1091-1093, 2009.

22. Bindea G, Galon J and Mlecnik B: CluePedia Cytoscape plugin: Pathway insights using integrated experimental and in silico data. Bioinformatics 29: 661-663,2013.

23. Huang da W, Sherman BT and Lempicki RA: Systematic and integrative analysis of large gene lists using DAVID bioinformatics resources. Nat Protoc 4: 44-57, 2009.

24. Huang da W, Sherman BT and Lempicki RA: Bioinformatics enrichment tools: Paths toward the comprehensive functional analysis of large gene lists. Nucleic Acids Res 37: 1-13, 2009.

25. Yao W, Wang X, Cai Q, Gao S, Wang J and Zhang P: Knockdown of TRAF4 expression suppresses osteosarcoma cell growth in vitro and in vivo. Int J Mol Med 34: 1655-1660, 2014

26. Yang $\mathrm{K}$, Wang $\mathrm{F}$ and Han JJ: TRAF4 promotes the growth and invasion of colon cancer through the Wnt/ $\beta$-catenin pathway. Int J Clin Exp Pathol 8: 1419-1426, 2015.

27. Yang J, Wei D, Wang W, Shen B, Xu S and Cao Y: TRAF4 enhances oral squamous cell carcinoma cell growth, invasion and migration by Wnt- $\beta$-catenin signaling pathway. Int J Clin Exp Pathol 8: 11837-11846, 2015

28. Zhu L, Zhang S, Huan X, Mei Y and Yang H: Down-regulation of TRAF4 targeting RSK4 inhibits proliferation, invasion and metastasis in breast cancer xenografts. Biochem Biophys Res Commun 500: 810-816, 2018

29. Chen L, Zhang R, Li P, Liu Y, Qin K, Fa ZQ, Liu YJ, Ke YQ and Jiang XD: P53-induced microRNA-107 inhibits proliferation of glioma cells and down-regulates the expression of CDK6 and Notch-2. Neurosci Lett 534: 327-332, 2013.

30. Chen L, Chen XR, Chen FF, Liu Y, Li P, Zhang R, Yan K, Yi YJ, Xu ZM and Jiang XD: MicroRNA-107 inhibits U87 glioma stem cells growth and invasion. Cell Mol Neurobiol 33: 651-657, 2013

31. He J, Zhang W, Zhou Q, Zhao T, Song Y, Chai L and Li Y: Low-expression of microRNA-107 inhibits cell apoptosis in glioma by upregulation of SALL4. Int J Biochem Cell Biol 45: 1962-1973, 2013.

32. Ji Y, Wei Y, Wang J, Ao Q, Gong K and Zuo H: Decreased expression of microRNA-107 predicts poorer prognosis in glioma. Tumour Biol 36: 4461-4466, 2015.

33. Wolter M, Werner T, Malzkorn B and Reifenberger G: Role of microRNAs located on chromosome arm 10q in malignant gliomas. Brain Pathol 26: 344-358, 2016.

34. Xu X, Cai N, Zhi T, Bao Z, Wang D, Liu Y, Jiang K, Fan L, Ji J and Liu N: MicroRNA-1179 inhibits glioblastoma cell proliferation and cell cycle progression via directly targeting E2F transcription factor 5. Am J Cancer Res 7: 1680-1692, 2017.

35. Wang J, Li J, Guo F and Yan Y: MicroRNA-133a inhibits the malignant behavior of glioma via downregulation of matrix metallopeptidase 9. Mol Med Rep 13: 3220-3226, 2016.

36. Sakr M, Takino T, Sabit H, Nakada M, Li Z and Sato H: miR-150-5p and miR-133a suppress glioma cell proliferation and migration through targeting membrane-type-1 matrix metalloproteinase. Gene 587: 155-162, 2016.

37. Wang J,Li Y and Jiang C: MiR-133b contributes to arsenic-induced apoptosis in U251 glioma cells by targeting the hERG channel. J Mol Neurosci 55: 985-994, 2015.

38. Li C, Liu Z, Yang K, Chen X, Zeng Y, Liu J, Li Z and Liu Y: miR-133b inhibits glioma cell proliferation and invasion by targeting Sirt1. Oncotarget 7: 36247-36254, 2016.

39. Artinian N, Cloninger C, Holmes B, Benavides-Serrato A, Bashir T and Gera J: Phosphorylation of the hippo pathway component AMOTL2 by the mTORC 2 kinase promotes YAP signaling, resulting in enhanced glioblastoma growth and invasiveness. J Biol Chem 290: 19387-19401, 2015.

40. Xu Y, Stamenkovic I and Yu Q: CD44 attenuates activation of the hippo signaling pathway and is a prime therapeutic target for glioblastoma. Cancer Res 70: 2455-2464, 2010.

41. Liu Y, Lu J, Zhang Z, Zhu L, Dong S, Guo G, Li R, Nan Y, Yu K, Zhong Y and Huang Q: Amlexanox, a selective inhibitor of IKBKE, generates anti-tumoral effects by disrupting the Hippo pathway in human glioblastoma cell lines. Cell Death Dis 8: e3022, 2017.

42. Lee Y, Lee JK, Ahn SH, Lee J and Nam DH: WNT signaling in glioblastoma and therapeutic opportunities. Lab Invest 96: $137-150,2016$ 
43. De Robertis A, Valensin S, Rossi M, Tunici P, Verani M, De Rosa A Giordano C, Varrone M, Nencini A, Pratelli C, et al: Identification and characterization of a small-molecule inhibitor of Wnt signaling in glioblastoma cells. Mol Cancer Ther 12: 1180-1189, 2013

44. Rathod SS, Rani SB, Khan M, Muzumdar D and Shiras A: Tumor suppressive miRNA-34a suppresses cell proliferation and tumor growth of glioma stem cells by targeting Akt and Wnt signaling pathways. FEBS Open Bio 4: 485-495, 2014.

45. Zhang W, Shen C, Li C, Yang G, Liu H, Chen X, Zhu D, Zou H, Zhen Y, Zhang D and Zhao S: miR-577 inhibits glioblastoma tumor growth via the Wnt signaling pathway. Mol Carcinog 55: $575-585,2016$.

46. Kahlert UD, Maciaczyk D, Doostkam S, Orr BA, Simons B, Bogiel T, Reithmeier T,Prinz M, Schubert J, Niedermann G, et al: Activation of canonical WNT/ $\beta$-catenin signaling enhances in vitro motility of glioblastoma cells by activation of ZEB1 and other activators of epithelial-to-mesenchymal transition. Cancer Lett 325: 42-53, 2012.

47. Kaur N, Chettiar S, Rathod S, Rath P, Muzumdar D, Shaikh ML and Shiras A: Wnt3a mediated activation of Wnt $/ \beta$-catenin signaling promotes tumor progression in glioblastoma. Mol Cell Neurosci 54: 44-57, 2013

48. Duan R, Han L, Wang Q, Wei J, Chen L, Zhang J, Kang C and Wang L: HOXA13 is a potential GBM diagnostic marker and promotes glioma invasion by activating the Wnt and TGF- $\beta$ pathways. Oncotarget 6: 27778-27793, 2015.

49. Kamino M, Kishida M, Kibe T, Ikoma K, Iijima M, Hirano H, Tokudome M, Chen L, Koriyama C, Yamada K, et al: Wnt-5a signaling is correlated with infiltrative activity in human glioma by inducing cellular migration and MMP-2. Cancer Sci 102: 540-548, 2011

50. Yu JM, Jun ES, Jung JS, Suh SY, Han JY, Kim JY, Kim KW and Jung JS: Role of Wnt5a in the proliferation of human glioblastoma cells. Cancer Lett 257: 172-181, 2007.
51. Hu B, Wang Q, Wang YA, Hua S, Sauvé CG, Ong D, Lan ZD, Chang Q, Ho YW, Monasterio MM, et al: Epigenetic Activation of WNT5A drives glioblastoma stem cell differentiation and invasive growth. Cell 167: 1281-1295.e18, 2016.

52. Bhat KP, Salazar KL, Balasubramaniyan V, Wani K, Heathcock L, Hollingsworth F, James JD, Gumin J, Diefes KL, Kim SH, et al: The transcriptional coactivator TAZ regulates mesenchymal differentiation in malignant glioma. Genes Dev 25: 2594-2609, 2011.

53. Yuan J, Xiao G, Peng G, Liu D, Wang Z, Liao Y, Liu Q, Wu M and Yuan X: MiRNA-125a-5p inhibits glioblastoma cell proliferation and promotes cell differentiation by targeting TAZ. Biochem Biophys Res Commun 457: 171-176, 2015.

54. Schiffgens S, Wilkens L, Brandes AA, Meier T, Franceschi E, Ermani M, Hartmann C, Sandalcioglu IE and Dumitru CA: Sex-specific clinicopathological significance of novel (Frizzled-7) and established (MGMT, IDH1) biomarkers in glioblastoma. Oncotarget 7: 55169-55180, 2016.

55. Wald JH, Hatakeyama J, Printsev I, Cuevas A, Fry WHD, Saldana MJ, VanderVorst K, Rowson-Hodel A, Angelastro JM, Sweeney C and Carraway KL Rd: Suppression of planar cell polarity signaling and migration in glioblastoma by Nrdp1-mediated Dvl polyubiquitination. Oncogene 36: 5158-5167, 2017.

56. Wang LL, Sun KX, Wu DD, Xiu YL, Chen X, Chen S, Zong ZH, Sang XB, Liu Y and Zhao Y: DLEU1 contributes to ovarian carcinoma tumourigenesis and development by interacting with miR-490-3p and altering CDK1 expression. J Cell Mol Med 21: 3055-3065, 2017.

57. Li X, Li Z, Liu Z, Xiao J, Yu S and Song Y: Long non-coding RNA DLEU1 predicts poor prognosis of gastric cancer and contributes to cell proliferation by epigenetically suppressing KLF2. Cancer Gene Ther 25: 58-67, 2018. 